\title{
The Prognostic Value of One-Step Nucleic Acid Amplification (OSNA) and Immunohistochemistry (IHC) in Ex Vivo Sentinel Lymph Nodes in Stage I \& II Colonic Cancer Patients - A Multicenter Cohort Study
}

\author{
RLA van der Linden ${ }^{1 *}$, JC van der Linden ${ }^{2}$, FJ Vogelaar ${ }^{3}$, DJ Lips ${ }^{1}$, CJH van de Velde ${ }^{4}$ and K Bosscha ${ }^{1}$ \\ ${ }^{1}$ Department of Surgery, Jeroen Bosch Hospital, Netherlands \\ ${ }^{2}$ Department of Pathology, Jeroen Bosch Hospital, Netherlands \\ ${ }^{3}$ Department of Surgery, Vie Curi Medical Center, Netherlands \\ ${ }^{4}$ Department of Surgery, Leiden University Medical Center, Netherlands
}

${ }^{*}$ Corresponding author: RLA van der Linden, Department of Surgery, Jeroen Bosch Hospital, 's-Hertogenbosch, the Netherlands

\begin{abstract}
Introduction: Sentinel lymph node (SLN) mapping with multilevel-sectioning and immunohistochemistry (IHC) or OneStep Nucleic Acid Amplification (OSNA) are considered promising techniques to improve LN staging in colon cancer. This study investigates the prognostic significance of OSNA- and IHC-analysis of ex vivo SNLs of stage I\&II colon cancer patients.

Methods: 86 stage I\&II colon cancer patients were identified from two previously conducted cohort studies. All SLNs were examined with Hematoxylin \& Eosin-staining (H\&E), multilevel-sectioning with IHC and OSNA analysis. Kaplan-Meier and coxregression analysis were performed to determine the prognostic significance.

Results: Mean disease-free survival (DFS) for OSNA-positive vs OSNA-negative patients was 62.5 vs 62.0 months ( $p=0.886$ ) and for IHC-positive vs IHC-negative patients 58.1 vs 62.9 months $(\mathrm{p}=0.519)$. Cox-regression analysis showed that positive OSNAstatus was not prognostic for DFS (hazard ratio $(\mathrm{HR})=0.89, \mathrm{p}=0.886)$. Additionally, also positive IHC-status showed no significant difference for DFS (HR 1.47, $\mathrm{p}=0.522$ ).

Conclusions: Additional OSNA or IHC-analysis of ex vivo SLNs does not provide a more accurate prognostic value in this small group of stage I\&II colon cancer patients compared with conventional H\&E. A bigger study is needed to set these results in a broader perspective. Further research is required to identify other clinically relevant upstaging techniques and prognostic factors to tailor the treatment plans of these patients.
\end{abstract}

\section{Introduction}

Colorectal cancer (CRC) is the third most common cancer worldwide with nearly 1,4 million newly diagnosed patients each year, accounting for $8 \%$ of all cancer related deaths [1,2]. In CRC, oncologic surgical resection is the primary therapy, followed by adjuvant chemotherapy on indication. Lymph node (LN) metastasis are one of the most important prognostic factors and are associated with worsened 5-year survival [3]. Previous studies demonstrated that $20-30 \%$ of node negative (stage I\&II) patients suffer from disease recurrence within 5 years after surgery [4,5]. This could

suggest that current conventional histopathological staging procedures, consisting of one or two sections of Hematoxylin \& Eosin (H\&E) through each detected LN, may not be precise enough to accurately describe the metastatic load in the resected LNs, possibly leading to undertreatment [6]. More accurate staging techniques are therefore needed to upstage colorectal patients considered to be node negative with conventional H\&E examination. In order to improve the detection of LN metastasis, different techniques have been investigated. Sentinel lymph node (SLN) mapping with multilevel-sectioning and immunohistochemistry 
(IHC) has been proposed as a promising technique for improved LN staging in patients with colorectal cancer [6,7]. Various studies have demonstrated that the use of either in-vivo or ex-vivo SLN mapping can achieve upstaging rates up to $35 \%$ in CRC patients [6, 7]. More recently, the use of One-Step Nucleic Acid Amplification (OSNA) has been trialed in patients with CRC [8-10]. This technique, which is already used in approximately 200 centers as an intra-operative diagnostic tool in breast cancer patients, uses the reversetranscription loop-mediated isothermal amplification (TR-LAMP) method for mRNA amplification [11]. OSNA determines, within 30 minutes, the metastatic status of a whole LN. In recent years, some groups have investigated OSNA in patients with CRC and described it as a reliable method for improved staging of lymphatic metastases in CRC, with reported upstaging rates of $20-25 \%[8,9]$.

The long-term prognostic significance of all these new ultrastaging and upstaging techniques in CRC patients has, until now, not yet been fully investigated. Gaining this information could be important as upstaging and ultra-staging could also lead to overtreatment. Therefore, it is of critical importance to study the long-term value of these techniques. The objective of this study is to investigate the prognostic value of OSNA and IHC in ex vivo detected SLNs of stage I\&II (H\&E node negative) colon cancer patients.

\section{Materials and methods}

\section{Patient Selection}

For this retrospective follow-up study, patients with stage I\&II colon cancer were identified from two previously conducted cohort studies [8]. Patients underwent standard oncological resection between November 2008 and December 2013 at two different centers, Jeroen Bosch hospital (JBH), 's Hertogenbosch and Leiden University Medical Center Leiden (LUMC). Stage III patients, T0 patients and high-risk stage I and II patients that had received adjuvant systemic chemotherapy (i.e pT4 tumor, poorly differentiated histology, presence of lymph vascular invasion, localized perforation and bowel obstruction) were excluded from this study. All other exclusion criteria were reported in the previous study [8].

\section{Sentinel Lymph Node Mapping Pathological Examination and OSNA-Procedure}

SLN mapping was performed ex-vivo within 15 minutes of resection. In patients operated at the JBH, SLN mapping was performed using Patent blue, whereas SLN mapping in the LUMC was performed using indocyanine green (ICG) and near-infrared light. The patent was injected submucosally and circumferentially around the tumor. In both centers the first three colored lymph nodes were marked as SLNs. The SLNs were processed in the same way in both hospitals as previously described [8]. In brief, the SLNs were cut into 4 pieces and labeled a, b, c and d. Slices a and $c$ were pooled together and analysed using OSNA, and slices b and $\mathrm{d}$ were subjected to an intensive multilevel sectioning histologic workup, cut into 4 levels with $250 \mu \mathrm{m}$ between each level, with H\&E and immunohistochemistry (anti-Pancytokeratin) [8]. The methods used for OSNA analysis are described in the instrument IFU and reagents package inserts [11,12]. Colon cancer was staged according to the 7th version of the TNM classification system [13]. The SLN's were examined for the presence of tumor cells. All lymph nodes free from metastasis were judged node negative. Sentinel lymph nodes with at least 1 observed tumor cell detected with immunohistochemistry or $>250$ mRNA copies/ $\mu \mathrm{L}$ in the OSNA analysis were judged as positive. A distinction in macro-metastasis [ $>2 \mathrm{~mm}$ ], micro-metastasis [ $>0.2$ and $\geq 2 \mathrm{~mm}$ ] and isolated tumor cells (ITC) $[\leq 0.2 \mathrm{~mm}]$ was made for H\&E and IHC [14]. Importantly, only results of the conventional H\&E staging were used in clinical practice for interdisciplinary tumor board decision making.

\section{Follow Up}

Follow up data were collected retrospectively from the hospital related clinical charts with a median follow up of 50 months. Disease free survival was defined as absence of local or distant metastatic disease diagnosed with the use of computed tomography, MRI or biopsy. Standard follow up examinations were performed using the Dutch oncological follow-up guidelines for colon cancer after curative colon cancer resection [15].

\section{Statistical Analysis}

Statistical analysis was performed using SPSS version 23 (SPSS: An IBM Company, Chicago IL). Continuous variables were compared with the Student's t, Mann-Whitney, one-way ANOVA and KruskalWallis tests, where appropriate. Differences among proportions derived from categorical data were compared using the Fischer's or the Pearson chi-square tests, where appropriate. All $p$ values in the univariate analyses were two-sided and considered statistically significant if $\mathrm{p} \leq 0.05$. Cumulative survival was calculated with the Kaplan-Meier function and comparison were performed with Log Rank test. The COX regression model was used to identify the adjusted hazard ratio of survival for patients with OSNA and IHC workup. Data are presented as mean (SD), median (IQR), and HR (95\% CI) where appropriate.

\section{Results}

\section{Baseline characteristic}

A total of 94 patients were included in the present analysis. Of the conventional $\mathrm{H} \& \mathrm{E}$ negative patients, in four patients there was no tumor present (T0) and to 4 node negative patients featuring high risk factors adjuvant systemic chemotherapy was given who were also excluded. Follow-up was successfully completed in all 86 patients with a median follow up of 50 months (SD:13,31). Baseline characteristics are shown in Table 1. 
Table 1: Baseline characteristics.

\begin{tabular}{|c|c|c|c|}
\hline & $\begin{array}{l}\text { Jeroen Bosch } \\
\text { Hospital }\end{array}$ & LUMC & P-value \\
\hline $\begin{array}{l}\text { Total number of } \\
\text { patients }(\mathrm{N})\end{array}$ & 67 & 19 & - \\
\hline \multicolumn{4}{|c|}{ Gender (\%) } \\
\hline Female & $30(34,6)$ & $8(9.3)$ & \multirow{2}{*}{ 0.836\# } \\
\hline Male & $37(43.0)$ & $11(12.8)$ & \\
\hline \multicolumn{4}{|c|}{ Resection type (\%) } \\
\hline Right hemi colectomy & $36(41.9)$ & $11(12.8)$ & \multirow{6}{*}{$0.441 \#$} \\
\hline Left hemi colectomy & $6(7.0)$ & $2(2.3)$ & \\
\hline Sigmoid resection & $22(25.6)$ & $4(4.7)$ & \\
\hline Subtotal colectomy & $1(1.2)$ & $0(0)$ & \\
\hline Total colectomy & $1(1.2)$ & $0(0)$ & \\
\hline Transversectomy & $1(1.2)$ & $2(2.3)$ & \\
\hline \multicolumn{4}{|c|}{ Approach (\%) } \\
\hline Laparotomy & $7(8.1)$ & $4(4.7)$ & \multirow{2}{*}{$0.222 \#$} \\
\hline Laparoscopic & $60(69.8)$ & $15(17.4 \%)$ & \\
\hline \multicolumn{4}{|c|}{ Pathological tumor stage (\%) } \\
\hline $\mathrm{T} 1$ & $3(3.5)$ & $3(3.4)$ & \multirow{4}{*}{$0.198 \#$} \\
\hline $\mathrm{T} 2$ & $18(20.9)$ & $6(7.0)$ & \\
\hline $\mathrm{T} 3$ & $45(52.3)$ & $9(10.5)$ & \\
\hline $\mathrm{T} 4$ & $1(1.2)$ & $1(1.2)$ & \\
\hline \multicolumn{4}{|c|}{ Pathological nodal stage (\%) } \\
\hline N0 & $67(77.9)$ & $19(22.1)$ & \\
\hline N1 & 0 & 0 & - \\
\hline $\mathrm{N} 2$ & 0 & 0 & \\
\hline$(.)=.\%, \#=$ Pearso & $\begin{array}{l}\text { hi-Square Asy } \\
\text { ersitair medisc }\end{array}$ & $\begin{array}{l}\text { (2-sided), LU } \\
\text { ntrum }\end{array}$ & Leids \\
\hline
\end{tabular}

\section{Oncological Follow Up}

During the follow-up period 16 patients died of whom three patients died due to cancer related progressive disease. In 10 patient's death was not cancer related - in four the cause of death was not further specified, one patient died due to postoperative complications, four dues to another malignancy and one patient due to a cerebro-vascular insult. In three patients the cause of death was unknown. Overall, the mean overall survival (OS) was 61.6 months. During the follow-up period 11 of $86(12.8 \%)$ pN0 patients had tumor recurrence. Overall, mean disease-free survival (DFS) was 62.2 months $(95 \% \mathrm{CI}=58.4-66.1$ months $)$. Distant recurrences were localized in the liver $(\mathrm{N}=6)$, lung $(\mathrm{N}=4)$, skin $(\mathrm{N}=1)$ and brain $(\mathrm{N}=1)$. Four patients had distant metastasis at two different places at time of diagnosis. Local regional recurrence was localized in lymph nodes $(\mathrm{N}=3)$.

\section{Prognostic Significance of OSNA and IHC in Ex Vivo SLN}

A total of 18 patients were node positive by OSNA analysis and 68 OSNA-negative. With the use of multilevel sectioning with immunohistochemistry (IHC) 30 patients were node positive and 54 IHC-negative. Two patients had missing IHC data. In the IHC node positive patients micro-metastases were found in 2 patients and isolated tumor cells in 28 patients. Recurrence occurred in 2 of OSNA-positive patients vs 9 of OSNA-negative patients and in 5 of IHC-positive patients vs 6 of IHC-negative patients (Table 2). No significant difference in mean DFS for OSNA-positive vs OSNAnegative patients was observed (62.5 months vs 62.0 months $(\mathrm{p}=0.886)$ ). Also mean DFS for IHC-positive vs IHC-negative patients showed no significant difference (58.1 months vs 62.9 months ( $p=0.519)$. Survival data is shown in Table 3 and figure $1 a, b$ \& $2 a, b$. The prognostic independence of OSNA and IHC was tested with cox regression analysis. A positive-OSNA status was not associated with significant shorter disease-free survival, (Hazard ratio $[\mathrm{HR}]=0.89$, $95 \% \mathrm{CI}=0.19-4.14, \mathrm{p}=0.886$ ). A positive-IHC status was also not associated with significant shorter disease-free survival, (Hazard ratio $[\mathrm{HR}]=1.47,95 \% \mathrm{CI}=0.45-4.83, \mathrm{p}=0.522$ ).

Table 2: Recurrence characteristics.

\begin{tabular}{|c|c|c|c|}
\hline \multicolumn{4}{|c|}{ Recurrence } \\
\hline & No & Yes & p-value \\
\hline \multicolumn{4}{|c|}{ OSNA (SLNs) } \\
\hline Positive & $16(18.6)$ & $2(2.3)$ & \multirow{3}{*}{$\mathrm{P}=0.886$} \\
\hline Negative & $59(68.6)$ & $9(10.5)$ & \\
\hline Total & 75 (87.2) & $11(12.8)$ & \\
\hline \multicolumn{4}{|c|}{ Fine pathology (SLNs) } \\
\hline Positive & 25 (29.1) & $5(5.8)$ & \multirow{4}{*}{$\mathrm{P}=0.659$} \\
\hline Negative & 48 (55.8) & $6(7.0)$ & \\
\hline Missing & $2(2.3)$ & 0 & \\
\hline Total & 75 (87.2) & $11(12.8)$ & \\
\hline
\end{tabular}

Table 3: Kaplan-Meier \& cox regression analysis.

\begin{tabular}{|c|c|c|c|}
\hline \multirow{2}{*}{$\begin{array}{c}\text { Kaplan-Meier } \\
\text { analysis }\end{array}$} & \multicolumn{3}{|c|}{ Disease free survival } \\
\hline & Estimate & $95 \% \mathrm{CI}$ & p-value \\
\hline $\begin{array}{l}\text { OSNA analysis } \\
\text { node positive }\end{array}$ & 62.5 & $54.9-70.0$ & \multirow{2}{*}{0.886} \\
\hline $\begin{array}{l}\text { OSNA analysis } \\
\text { node negative }\end{array}$ & 62.0 & $57.6-66.3$ & \\
\hline $\begin{array}{l}\text { IHC node } \\
\text { positive }\end{array}$ & 58.1 & $51.6-64.6$ & \multirow{2}{*}{0.519} \\
\hline $\begin{array}{l}\text { IHC node } \\
\text { negative }\end{array}$ & 62.9 & $58.2-67.7$ & \\
\hline
\end{tabular}

\begin{tabular}{|c|c|c|c|}
\hline \multirow{2}{*}{ Cox regression } & HR & $\mathbf{9 5 \%}$ CI & p-value \\
\cline { 2 - 4 } & 0.89 & $0.19-4.14$ & 0.886 \\
\hline OSNA analysis & 1.47 & $0.45-4.83$ & 0.522 \\
\hline IHC analysis & \multicolumn{3}{|c|}{0.05} \\
\hline \multicolumn{3}{|c|}{ HR: hazard ratio, 95\%CI= confidence interval, P= significance level < } \\
\hline
\end{tabular}




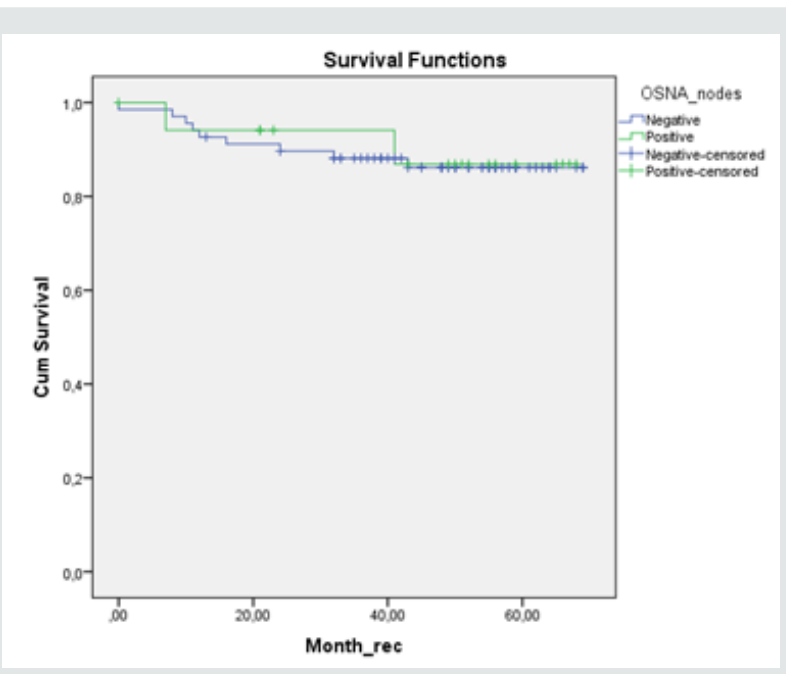

Figure 1a: Kaplan-Meier analysis of DFS with the use of OSNA staging.

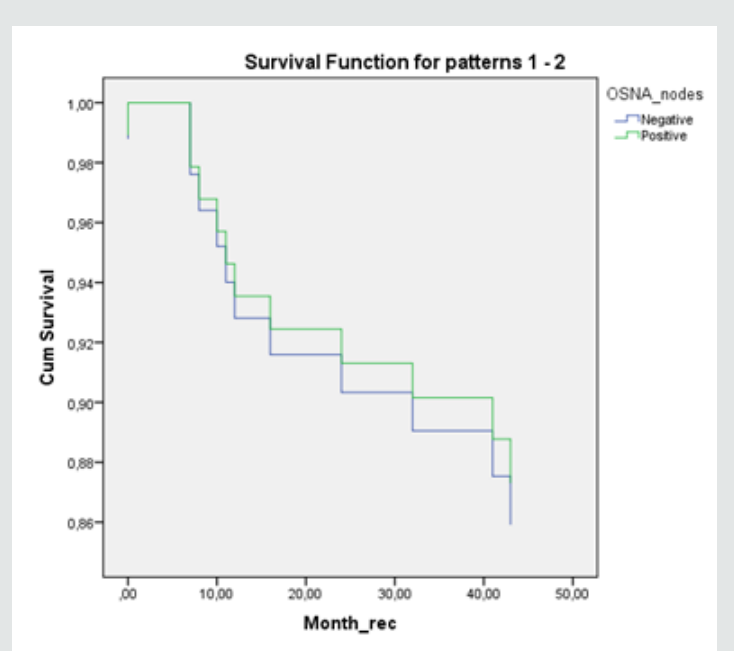

Figure 1b: Cox regression analysis of DFS with the use of OSNA staging.

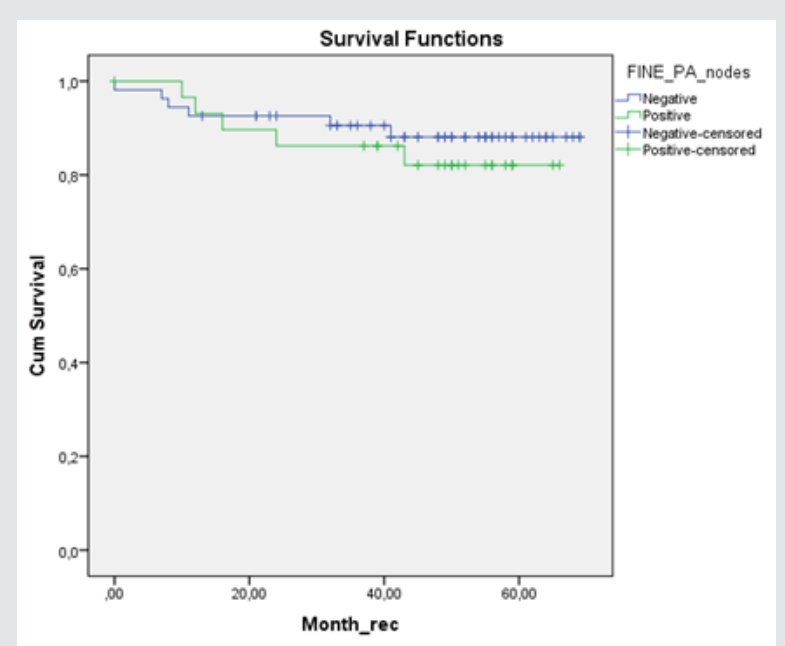

Figure 2a: Kaplan-Meier analysis of DFS with the use of IHC staging.

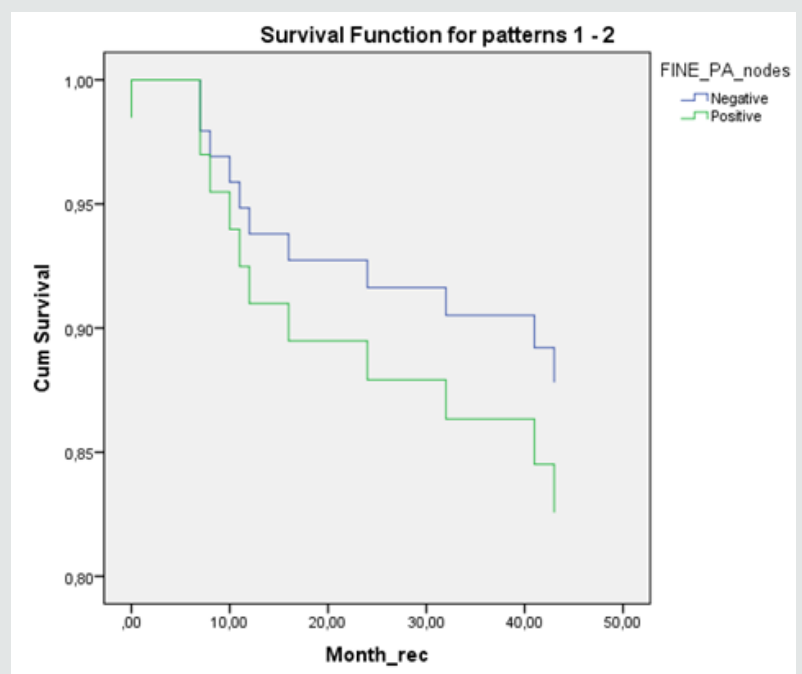

Figure 2b: Cox regression analysis of DFS with the use of IHC staging.

\section{Discussion}

Our study is one of the first published study investigating the prognostic value for disease free survival of OSNA in the long-term follow-up of ex vivo detected SLNs of a small group of stage I\&II colon cancer patients, showing no additional prognostic value. Whilst OSNA analysis and IHC can lead to upstaging of early colon cancer patients as described by Vogelaar et al, positive OSNA or IHC status of the ex vivo detected SNLs were no independent predictors for disease free survival in stage I\&II colon cancer patients [8]. Therefore, these new upstaging techniques does not provide a more accurate prognostic value. However, the strength of evidence of these findings might be limited because of the reduced power of this study, since this study consisted of a small group of patients. Therefore, the value of these findings must be proven by a bigger study to set these results in a broader perspective. On the contrary, just recently, Itabashi et al. published a 3-years follow-up study of stage I-III CRC patients with sub-analyses in a small group of 70 stage II patients. Showing a significant worse prognosis for stage II OSNA-positive patients [16]. However, 55\% of the OSNA-positive and $22 \%$ of the OSNA-negative patients were treated with chemotherapy. Since we corrected for this confounder the results of both studies are not comparable. Furthermore, we believe that our study demonstrates more representative results since chemotherapy influence survival outcome. The above shown discrepancy in OSNA-positive SLNs and SLNs harboring micro metastasis with IHC suggests that the OSNA cut-off value for micro metastasis ( $>250-5000$ copies of mRNA) is maybe not accurate enough to make a prognostic distinction between positive and negative LN status in stage I\&II colon cancer patients [11-17]. New studies in breast cancer patients showed a more accurate cut-off value ( $>2500$ copies of mRNA) for the detection of micro metastases as a prognostic cut-off value, supporting this suggestion $[17,18]$. Therefore, it is likely that some LNs harboring ITCs are staged as 
positive nodes with the OSNA analysis leading to upstaging without clinical prognostic relevance, which is in line with some previous studies showing the prognostic value of micro metastasis but not of ITCs [4-20]. The strength of evidence of this study might be limited because of the combination of datasets from two different hospitals. However, both hospitals used the same protocol for the work up of the LNs and OSNA analysis. Although the type of marker used to detect the SLNs was different between the two hospitals, previous studies have shown that there is a high correlation between these different makers, and therefore it is very likely that this difference did not affect the detection rates [21]. Besides the OSNA cut-off value, the prognostic value of total tumor load (TTL) is recently investigated in breast cancer patients [22]. TTL is defined as the total number of CK19 mRNA in all OSNA positive SLNs [22]. These studies demonstrated that a TTL of $>15.000$ mRNA is a new tool to predict the metastatic involvement of non-sentinel axillary nodes [22]. This new intra-operative tool could be of interest in early colon cancer patients when, in the future, more minimally invasive surgery will be performed. However, this data is not yet available in colon cancer patients and therefore further research is needed.

Furthermore, it is noteworthy thatalmost all disease recurrences seem to be of haematogenic nature. This metastatic pattern, the knowledge that $20-30 \%$ of stage I\&II patients suffer from disease recurrence within 5 years after surgery and above mentioned results could suggest that LN assessment has less clinical value in stage I\&II CRC patients than expected $[4,5]$. Another alternative that has been investigated in recent years is the prognostic value of tumor specific histopathological characteristics in these patients. Some of these characteristics of interest are tumor budding, poorly differentiated clusters and peri-neural invasion. Literature regarding these tumor specific histopathological characteristics is not unambiguously, but seems to have prognostic value in defining high risk stage I\&II CRC patients [23,24]. Besides histopathological characteristics, several molecular biomarkers for stage I\&II CRC patients have been studied in recent years, of which microsatellite instability (MSI) and mutations in the KRAS, BRAF and PIK3CA genes are the most wellknown biomarkers [25]. The prognostic value of these biomarkers in stage I\&II CRC patients is not yet determined. However, recent studies show that these biomarkers might be valuable in staging I\&II CRC patients [25]. Further research is therefore necessary to identify other clinically relevant upstaging techniques or molecular biomarkers and histopathological prognostic factors to improve the treatment of stage I\&II CRC patients. In conclusion, this study demonstrated that OSNA-and IHC-analysis of ex vivo detected SLNs does not provide a more accurate prognostic value for disease free survival in a small group of patients with non-metastasized colon cancer compared with the conventional H\&E. However, a bigger study is needed to set these results in a broader perspective. Further research is required to identify other clinically relevant ultra-staging and upstaging techniques or molecular biomarkers and histopathological prognostic factors to identify high risk stage I\&II CRC patients, to improve the prognostic assessments and tailor the treatment plans.

\section{Acknowledgement}

The authors thank Sysmex Corporation for technical support and providing study material. M. Hilbink for statistical advice, J. Damen for technical support and B. Weixler for collecting the LUMC follow-up data.

\section{Statement of Ethics and Funding Sources}

The approval of the medical ethics committee of the participating hospitals were described in detail in the previous publication. According to this committees written informed consent was not necessary as this study concerned residual material and there were no treatment consequences. This study was performed according to the code of conduct for responsible use. Sysmex corporation provided the OSNA machine and study material for the OSNA analyses. This source had no role in the preparation of data or the manuscript.

\section{References}

1. Center MM, Jemalc A, Smith RA (2009) World wide variation in colorectal cancer. CA cancer J clin 59(6): 366-378.

2. Siegel RL, Miller, KD, Jemal A (2015) Cancer statistics. CA Cancer J Clin 65(1): 5-29.

3. Koebrugge B, Vogelaar FJ, Lips DJ, Pruijt JF, van der Linden JC, et al. (2011) The number of high-risk factors is related to outcome in stage II colonic cancer patients. Eur J Surg Oncol 37(11): 964-970.

4. Lips DJ, Koebrugge B, Liefers GJ, van de Linden JC, Smit VT, et al. (2011) The influence of micrometastases on prognosis and survival in stage I-II colon cancer patients: the Enroute plus sign in circle Study. BMC Surg 11: 11.

5. Sloothaak DAM, van der Linden RLA, van de Velden CJH, Bemelman WA4, Lips DJ, et al. (2017) Prognostic implications of occult nodal tumour cells in stage I and II colon cancer: The correlation between micro metastasis and disease recurrence. Eur J Surg Oncol 43(8): 1456-1462.

6. Smith J, Hwang H, Wiseman KW, Filipenko D, Phang PT (2006) Ex vivo sentinel lymph node mapping in colon cancer: improving the accuracy of pathologic staging? Am J Surg 191(5): 665-658.

7. Cahill RA, Leroy J, Marescaux J (2008) Could lymphatic mapping and sentinel node biopsy provide oncological providence for local resectional techniques for colon cancer? A review of the literature. BMC Surg 8: 17.

8. Vogelaar FJ, Reimers MS, van der Linden RLA, van der Linden JC, Smit VT, et al. (2014) The diagnostic value of One-Step nucleic acid Amplification (OSNA) for Sentinel Lymph Nodes in Colon Cancer Patients. Ann Surg Oncol. 21: 3924-3930.

9. Wild JB, Iqbal N, Francombe J, Papettas T2, Sanders DS, et al. (2017) Is it time for one-step nucleic acid amplification (OSNA) in colorectal cancer? A systematic review and meta-analysis. Tech Coloproctol 21(9): 693-699.

10. Shi F, Zhang Q Liang Z, Zhang M, Liu X (2017) One-step nucleic acid amplification assay is an accurate technique for sentinel lymph node biopsy of breast cancer patients: a meta-analysis. BJC 177(8): 11851191. 
11. Notomi T, Okayama H, Masubuchi H (2000) Loop-mediated isothermal amplification of DNA [serial online]. Nucleic Acids Res 28(12): E63.

12. 2010 Sysmex RD-100i training Guide, Netherlands.

13. American Cancer Society, AJCC, $7^{\text {th }}$ edition [serial online].

14. Hermanek P, Hutter RV, Sobin LH, etal (1999) International Union Against Cancer. Classification of isolated tumor cells and micrometastasis. Cancer 86(12): 2668-2673.

15. IKNL Dutch Guideline for colorectal cancer, Netherlands.

16. Itabashi M, Yamamoto H, Tomita N, Inomata M, Murata K, et al. (2019) Lymph node positivity in one-step nucleic acid amplification is a prognostic factor for postoperative cancer recurrence in patients with stage II colorectal cancer: A prospective, multicenter study. Ann Surg Oncol 27(4): 1077-1083.

17. Tsujimoto M, Nakabayashi K, Yoshidome K, Kaneko T, Iwase T, et al. (2007) One-step nucleic acid amplification for intraoperative detection of lymph node metastasis in breast cancer patients. Clin Cancer Res 13(16): 4807-4816.

18. Terrenato I, D’alicandro V, Casini B, Perracchio L, Rollo F, et al. (2017) A cut-off of 2150 cytokeratin 19 mRNA copy number in sentinel lymph node may be a powerful predictor of non-sentinel lymph node status in breast cancer patients. Plos one 12(2): e171517.
19. Faerden AE, SJo OH, Bukholm IR, Andersen SN, Svindland A, et al. (2011) Lymph node metastases and isolated tumor cells influence survival in stage I and II colon cancer. Dis Colon Rectum 54(2): 200-206.

20. Lee MR, Hong CW, Yoon SN, Lim SB, Park KJ, et al. (2006) Isolated tumor cells in lymph nodes are not prognostic marker for patients with stage I and II colorectal cancer. J Surg Oncol 93(1): 13-18.

21. Schaafsma BE, Verbeek FP, van der Vorst JR, Hutteman M, Kuppen PJ, et al. (2013) Ex vivo sentinel node mapping in colon cancer combining blue dye staining and fluorescence imaging. J Surg Re 183(1): 253-257.

22. Peg V, Espinosa-Bravo M, Vieites B, Vilardell F, Antúnez JR, et al. (2013) Intraoperative molecular analysis of total tumor load in sentinel lymph node: a new predictor of axillary status in early breast cancer patient. Breast Cancer Res Treat 139(1): 87-93.

23. Zhou Y, Wang H, Gong H, Cao M, Zhang G, et al. (2015) Clinical significance of perineural invasion in stage II-III colorectal cancer. Pathol Res Pract 221(11): 839-844.

24. Rogers AC, Winter DC, Heeney A, Gibbons D, Lugli A et al. (2016) Systematic review and meta-analysis of the impact of tumour budding in colorectal cancer. Br J Cancer. 115: 831-840.

25. Vogelaar FJ, Erning FNV, Reimers MS, Linden HV6, Pruijt H, et al. (2016) The prognostic value of microsatellite instability, KRAS, BRAF and PIK3CA mutations in stage II colon cancer patients. Mol Med 21(1): 1038-1046.

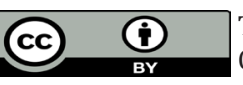

This work is licensed under Creative Commons Attribution 4.0 License

To Submit Your Article Click Here: Submit Article

DOI: $10.32474 /$ OAJOM.2020.03.000169

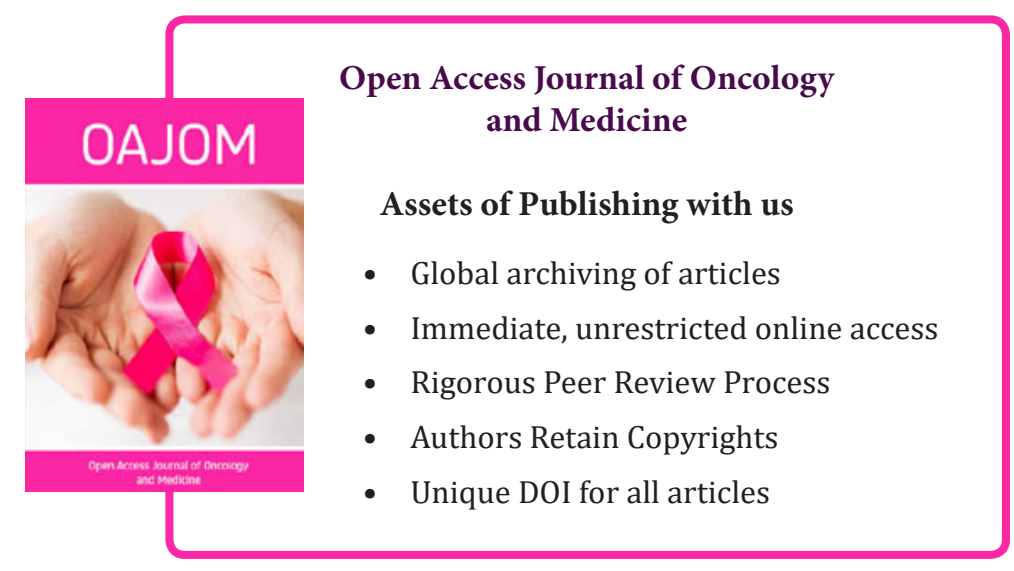

